\section{Printing in Medicine}

\section{In brief}

- $\quad 3 D$ printing is already being used for making artificial limbs, dental implants and other medical items specifically adapted to individual patients.

- Replacement organs cannot be produced yet and remain an option for the future.

- Access and intellectual property rights, data handling and protection, and incorporating additive manufacturing into medical practice represent crucial challenges.

\section{What is it about?}

Customised medical products from a 3D printer that restore or support bodily functions promise greater efficacy and functionality, lower costs and new options for therapy.

In 3D printing (additive manufacturing, AM, or rapid prototyping), parts with complex shapes can be created from different materials by means of computer-aided design. The product is constructed in a 3D printer by joining or solidifying layers of material rather than a blank being milled. This allows for single parts or small batches of parts to be manufactured very economically, requiring fewer tools and materials. Also, products can be manufactured almost everywhere in the world, even at home (do-it-yourself, DIY).

State of the art: 3D printing technologies are applied in different medical settings and are already state of the art in dentistry. Despite progress, many technical challenges remain, e.g. with regard to the generation and processing of data or growing biological tissue. Creating whole organs in a 3D printer from lab-grown cells is still at an experimental stage. In addition, some materials have insufficient properties or the objects' surface is too rough.

$3 \mathrm{D}$ printing is already being applied, and has often become economically important, in the following medical fields:
Prostheses and orthoses: Using data derived from the photographic measurement of single body parts, 3D printing allows for the manufacture of custom-fit artificial limbs (prostheses) and external supporting devices (orthoses). Data files can be easily sent across the world, ensuring that patients in developing countries can also benefit. Prostheses printed with 3D technology have greater likeness to natural limbs or exhibit greater functionality. Implants can be fabricated in advance with custom fit rather than having to be adapted during the operation.

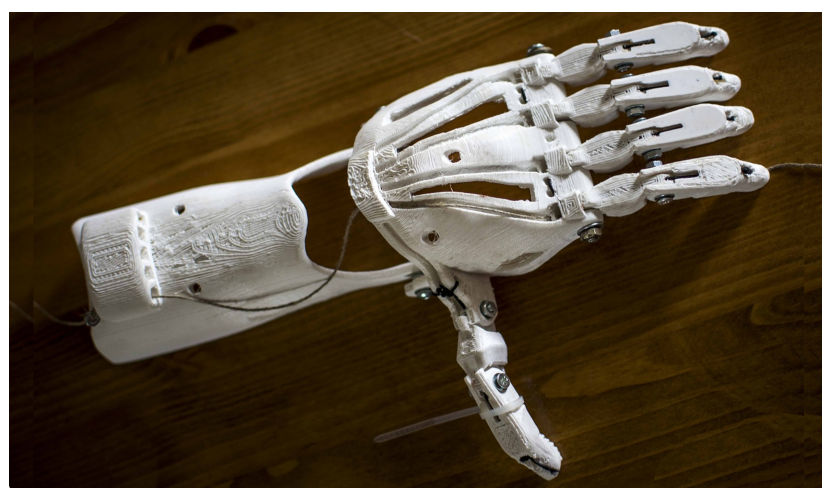

Who will guarantee the safety of 3D-printed artificial limbs from decentralised production?

Surgical models and educational tools: Customised surgical jigs from a 3D printer allow for easier and shorter operations. Thanks to precise models of patients' organs, operations can be planned, discussed and simulated.

Digital dental prostheses and hearing aids: Hearing aid shells made in a 3D printer have become the norm. 3Dprinted dental implants, too, have become common. Rapid prototyping, automation of the entire process chain and the decentralisation of the production saves time and costs.

Drug delivery: AM allows customised drug delivery by combining a patient-specific mix of drugs being printed into one single pill. Individually-designed and adapted implants release drugs in a targeted way.

Organ printing/tissue engineering: In the future, it will be possible to print skin or cartilage with 3D technology, but tissue engineering will mostly remain a research tool, for example for toxicity tests. 3D printing of entire organs to suit the need for donor organs is still not quite possible.

Progress in medical 3D printing not only opens up new possibilities for research and for therapy; it also has consequences for our society.

\section{Basic data}

Project title: Additive Manufacturing -

3D Printing in Medicine

Project team: $\quad$ KIT, Rathenau, DBT, ITA, a.o.

Duration: 10/2016-01/2018

Funded by: STOA/European Parliament 


\section{Far-reaching consequences}

Safety: Some plastic raw materials are toxic; biological materials pose special risks. 3D printing allows for decentralised manufacturing which makes centralised safety testing impossible. How product safety can be ensured under such circumstances remains unclear.

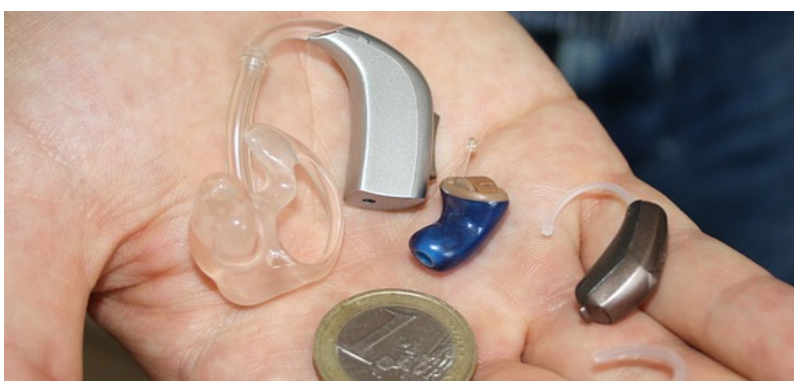

All production of hearing aid shells has turned to 3D printing.

Property rights and privacy: Medical data are subject to intellectual property rights (IPR). Sharing data for 3D printing (e.g. among DIY communities) challenges not only privacy but also IPR practices.

Ethical aspects: 3D-printed organs may one day reduce or replace animal testing and satisfy the need for human donor organs. Even if feasible, printing entire functional organs will always remain time-consuming and costly.

Access: Customised prostheses may become more readily available also in remote areas. Whether patients can access them will depend mostly on solving IPR questions.

Environmental issues: Compared to traditional milling, $3 \mathrm{D}$ printing requires fewer materials and tools, and less water. However, some raw materials for 3D printing are toxic.

Impact on jobs: 3D printing could worsen job prospects for, e.g., dental technicians. On the other hand, it might also lead to novel products and other types of jobs. Nevertheless, specialisation, division of labour and remote manufacturing will also increase in those jobs that already rely upon professional skills today (such as fabricating prostheses).

Therapy/enhancement: 3D printing for cosmetic purposes is already being used in dentistry. By contrast, high expectations, e.g. regarding organ printing for transplantations, have not yet been met. This could result in reduced interest and lead to a decrease in research efforts.

Economic aspects: In 2016, the worldwide share of 3D printing in all fields of industrial production was $11 \%$, of which one tenth being medical applications, with high growth rates. Some areas (hearing aids) have fully turned to $3 \mathrm{D}$ printing. An aging population and the trend to personalised medicine speed up the development. Although savings might be possible, customised high-tech products are costly and will remain so for the foreseeable future.

\section{What to do?}

Because medical 3D printing technology is inherently versatile, can be adapted to individual cases and applied regardless of location, and depends on computerised files, many challenges arise, requiring political action. These challenges can be addressed in different ways:

- Regulation: Many regulations in the different medical fields affected by 3D printing need to be adapted. One option is to develop a consistent framework for the technology. This would ease access and enhance safety and liability whilst preventing medical tourism. Adapting existing regulations in a case-specific way would be another option. However, whilst saving time and costs, grey areas and uncertainty would remain.

- Distribution of benefits: Innovation policy focussed on societal objectives could support projects addressing diseases with high social burdens and help small and medium-sized enterprises (SMEs) developing niche applications. Another option would be open competitive innovation policy that focusses on production costs. In both cases, open access/open data policy could be pursued and patents restricted to end user products.

- Participation: Public events could address societal values and expectations, and help to align technology development with societal preferences. Another option would be funding grassroot and/or DIY initiatives. In makerspaces, young people and other groups could gain new perspectives and exchange know-how.

\section{Further reading}

Ferrari et al. (2018) Additive bio-manufacturing: 3D printing for medical recovery and human enhancement. European Parliament - STOA Science and Technology Options Assessment

europarl.europa.eu/stoa/en/document/EPRS IDA(2018)614571

\section{Contact}

Helge Torgersen

Email: tamail@oeaw.ac.at

Phone: +43(1)51581-6582

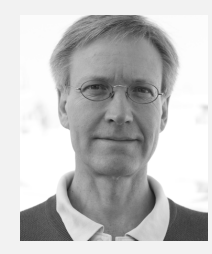

IMPRINT: Owner: Austrian Academy of Sciences; Legal person under public law (BGBI. 569/1921 i.d.F. BGBI. I 130/2003); Dr. Ignaz Seipel-Platz 2, A-1010 Vienna; Editor: Institute of Technology Assessment (ITA); Apostelgasse 23, A-1030 Vienna; www. oeaw.ac.at/ita/en. | Frequency: The ITA Dossiers are published irregularly and publish the research results of the ITA. The ITA Dossiers are published in print in small numbers only and are made available open access to the public via the Internet portal "epub.oeaw": epub.oeaw.ac.at/ita/ita-dossiers | ISSN: 2306-1960 | This work is licensed under a Creative Commons Attribution 4.0 International License: creativecommons.org/licenses/by/4.0/| ITA Dossier no. 39en, July 2018 | epub.oeaw.ac.at/ita/ita-dossiers/ita-dossier039en.pdf | c) (i) 